\title{
Morality not markets: a manifesto for the NHS; Response to Pollock, Frith, and Cox
}

\author{
Emma Clarke $^{1}$, Laurence Leaver ${ }^{1}$, Barnabas J Gilbert ${ }^{2^{*}}$
}

\author{
*Correspondence to: Barnabas J Gilbert, Email: bgilbert@g.harvard.edu \\ Copyright: $\odot 2015$ by Kerman University of Medical Sciences \\ Citation: Clarke E, Leaver L, Gilbert BJ. Morality not markets: a manifesto for the \\ NHS; Response to Pollock, Frith, and Cox. Int J Health Policy Manag 2015; 4: 327-328. \\ doi: 10.15171/ijhpm.2015.78 \\ Received: 20 March 2015, Accepted: 3 April 2015, ePublished: 4 April 2015
}

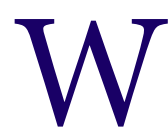

e would like to thank Pollock (1), Frith (2) and Cox (3) for their interesting analyses and suggestions on our article "Morality and markets in the NHS". A number of important themes arise: the lack of either practicality or morality of a healthcare market or privatisation; the need for values to be supported (where possible) by legislation; and the crucial importance of humanity and compassion in healthcare.

Healthcare will be a key issue in the upcoming UK general election. Practitioners frequently distance themselves from politics, concentrating instead on their primary duty to individual patients. However, it is increasingly relevant that the doctors of 2015 recognise their duty to society, as well as to the individual. From the advent of General Practitioner (GP) fundholding in the 1990s to the latest Clinical Commissioning Groups, rationing by healthcare professionals on behalf of the government has become more explicit. In balancing their duties to individuals, communities and society, doctors should be encouraged to speak out about issues that influence patient care. This should not be confined to whistleblowing, but should include engagement in the broader political process. The need for doctors to speak out in this way is heightened by what Allyson Pollock describes as the "managed decline" of the National Health Service (NHS). As she reiterates, "The NHS will last as long as there are folk left with the faith to fight for it". Despite continual frustrations with the political process, doctors, amongst others, are well-placed to understand many of these issues and to defend the NHS.

We applaud Pollock for making an explicitly political response to our article. If universal healthcare is to remain, it should be enshrined in law, which would require reinstatement of the legal obligation placed on the Secretary of State to provide healthcare, either through repeal of the Health and Social Care Act or through new or revised legislation which reinstates this fundamental principal of responsibility. Pollock emphasises the importance of what is set in law and statute as being the framework from which everything else is derived.

This principle can be applied to Frith's comments about the NHS Constitution. In response to our suggestion of a valuesexplicit approach, Frith suggests that this already exists, at least theoretically, in the form of the NHS Constitution. The Constitution reiterates the intended purposes of the
NHS, as a publicly accountable, comprehensive service available to all according to need, and makes explicit its values and commitments. However, Frith asks: "How useful is the Constitution in practice? How is compliance with the Constitution to be policed?... What does 'have regard' to the constitution actually mean...?" (1). One could also question the extent to which the NHS Constitution succeeds in placing reciprocal responsibilities on the public to make best use of the NHS (or to defend it).

The Health and Social Care Act was passed without a mandate, described by Pollock as "lacking in any meaningful [public] consultation". This undermines the central message of the NHS Constitution, that "the NHS belongs to us all" (4). The duty to "have regard" for the NHS Constitution, which was set out in the Health Act 2009 and extended by the Health and Social Care Act in 2012, has clearly been flouted, damaging the utility of the NHS Constitution. As we have touched upon, the Act further undermined the Constitution by removing the obligation of nationwide healthcare provision by the Secretary of State, which may further increase inequalities in provision. We agree that repeal of the Health and Social Care Act may help to enshrine morality in law, as proposed by Pollock, and would also improve congruence between the message of the Constitution thrown into question by Frith and the values underlying the operation of the NHS. In practice, owing in part to the nuances of the political process and in part to a desire to uphold important and positive aspects of this legislation (for example, greater involvement of clinicians in decisions about local commissioning, and an attempt to improve integration between health and social services), new or revised legislation may be a more likely and sustainable outcome.

All three authors agree that there are "moral limits of markets" (5) and that their application to healthcare is ethically wrong. Frith highlights twin forces at play, both of which are exacerbated by the Health and Social Care Act the use of market forces and privatisation. She explores the profound ethical implications of the latter, using the example of the privately run Hinchingbrooke Hospital. She emphasises that patient care is threatened both by conflicts of interest and by a lack of long-term accountability within the private sector. In addition, both Pollock and Frith highlight practical problems that stem from the use of markets in healthcare, including detrimental effects on cost effectiveness, inequality of provision and fragmentation of services. Frith explains that the principal justification for a healthcare market, namely its ability to drive up quality while reducing costs, is misconstrued, and Pollock agrees that healthcare markets 
have a negative impact on both quality and cost, associated, for example, with increasing contractual complexity.

As highlighted by Pollock, one of the results of the application of market forces to healthcare has been the development of performance measurement systems to increase competition. There is a problem in this approach; it implies that everything that counts can be counted and that everything that is counted counts. One of the challenges in improving care quality is to discover the best way to define and measure it. The Donabedian model (6) provides a framework for the evaluation of healthcare quality according to three categories: structure, process and outcomes. Process, including the interactions between patients and healthcare professionals, may well be the best measure of care quality, but is hardest to measure and most frequently ignored in preference of structure or outcomes.

At the heart of the care process is the practitioner-patient relationship, a vastly neglected area. John Cox is absolutely right to emphasise the importance of compassion. Emotional intelligence and empathy are necessary but not sufficient for true compassion; practitioners must also 'care' to the extent of being altruistic at some level, and to be willing to 'suffer alongside' (one literal derivation of 'compassion'). Cox talks about the quality of relationships between staff members and patients and how these relationships contribute to a culture of care. The very nature of these processes - which makes them difficult to measure - also makes them difficult to legislate. It is hard to imagine how such personal values could be enshrined in law, and indeed a contractual obligation to 'be compassionate' could negate the altruistic component of each practitioner's 'social gift' of caring, but this does not mean that such values are ephemeral. Indeed, as Cox floridly suggests: "watering the roots of compassion by politicians, patients, managers, faith communities and grass roots activists is more likely to yield the green shoots of hope in a very parched land". We agree with Cox that the cultural, religious and humanistic roots of caring deserve further attention. There has been increasing attention to communication skills in the training of healthcare professionals in recent years. However, the extension of good communication into empathy and compassion cannot be assumed; indeed, their exploration is frequently overlooked. Going beyond communication skills to examine the extent to which practitioners are humane (and why they choose to be) should be fruitful. How much should practitioners share of themselves with patients, for example? In "Practitioners and practices: a conflict of values", Julian Pratt discusses some of the different roles that practitioners juggle: the traditional biomedical model; the biographical role (as witness to a patient's life events); the role as carer; and, most poignantly perhaps, the role as healer (7). It is this latter role which has a spiritual dimension and an understanding of this role may be what moves some practitioners beyond empathy and towards genuine compassion.

As the NHS and its workers are squeezed from all angles, it is unsurprising that these values are sometimes lost. Repeal of the Health and Social Care Act may ease some of the pressures that have resulted from the increasing use of market forces - such as the focus on outcome-based performance measures - to allow refocus and rebuilding of a culture of caring.

In summary, we agree that the next Government should consider repealing or revising the Health and Social Care Act with respect to the Secretary of State's responsibility for national healthcare, and that the role of market forces and privatisation within the NHS should be more carefully critiqued. We believe that there are better and more appropriate ways to improve quality of care, starting with the gradual reversal of what Frith describes as "creeping marketisation and privatisation", which has been engrained in UK health policy since 1979. Of note, quality should not be reduced to numbers on a spreadsheet, ignoring fundamental aspects of care such as where practitioners position themselves in relationships with patients and whether they adopt a holistic approach. In understanding such complexities, the ethical dimension to healthcare has never been more important.

Ethical issues

Not applicable.

Competing interests

Authors declare that they have no competing interests.

Authors' contributions

All authors contributed to the concept and planning of the article. EC drafted the initial manuscript which was revised by LL and BJG. All authors agreed upon the final manuscript.

Authors' affiliations

${ }^{1}$ Green Templeton College, University of Oxford, Oxford, UK. ${ }^{2}$ Harvard University, Cambridge, MA, USA.

\section{References}

1. Pollock AM. Morality and values in support of universal healthcare must be enshrined in law; Comment on "Morality and Markets in the NHS". Int J Health Policy Manag 2015; forthcoming. doi: 10.15171/ijhpm.2015.61

2. Frith L. The Changing National Health Service: Market-Based Reform and Morality; Comment on "Morality and Markets in the NHS". Int J Health Policy Manag 2015; 4: 253-5. doi: 10.15171/ ijhpm.2015.39

3. Cox J. NHS values, compassion and quality indicators for relationship based person-centred healthcare; Comment on "Morality and markets in the NHS". Int J Health Policy Manag 2015; forthcoming. doi: 10.15171/ijhpm.2015.70

4. Department of Health (DOH). The NHS Constitution. Last updated Feb 2015. Available from: https://www.gov.uk/government/ uploads/system/uploads/attachment_data/file/170656/NHS_ Constitution.pdf

5. Sandel MJ. What Money Can't Buy: The Moral Limits of Markets. London: Allen Lane; 2012.

6. Kaionius PJ, Kazemi A. Structure and process quality as predictors of satisfaction with elderly care. Health Soc Care Community 2015; forthcoming. doi: 10.1111/hsc.12230

7. Pratt J. Practitioners and Practices a Conflict of Values? Oxford: Radcliffe Medical Press; 1995. 